\title{
Cinética de fermentación y degradabilidad ruminal in vitro de dietas con diferente fuente de nitrógeno
}

\section{Fermentation kinetics and ruminal degradability in vitro of diets with different nitrogen source}

\author{
${ }^{\bullet}$ Carlos Aguirre Valverde ${ }^{1}$, Marlene Medina VIllacís ${ }^{2}$, León Montenegro Vivas ${ }^{1}$, Adolfo Sánchez Laiño ${ }^{1}$ \\ Alexandra Barrera-Alvárez ${ }^{1}$, Italo Espinoza Guerra ${ }^{1}$ \\ ${ }^{1}$ Universidad Técnica Estatal de Quevedo. Facultad de Ciencias Pecuarias. Carrera de Ingeniería Zootécnica. \\ Campus Finca Experimental "La Maria”. CP. 121250. Km. 7 11/2 vía El Empalme, cantón Mocache. Los Ríos. Ecuador. \\ `caguirre@uteq.edu.ec; lmontenegro@uteq.edu.ec; arsanchez@uteq.edu.ec; barreraalvarez@yahoo.com; \\ iespinoza@uteq.edu.ec \\ ${ }^{2}$ Universidad Técnica Estatal de Quevedo. Facultad de Ciencias de la Ingeniería. Carrera de Ingeniería Agrondustrial. Campus \\ Ing. Manuel Haz Álvarez, km 1.5 vía a Santo Domingo de los Tsáchilas. EC.120501.Quevedo, Ecuador. mmedina@uteq.edu.ec
}

Resumen

$E_{\mathrm{de}}^{1}$ objetivo de este trabajo fue determinar la cinética de fermentación y degradabilidad ruminal in vitro de dietas con diferente fuente de nitrógeno para alimentación de rumiantes. Se formularon tres dietas (D) con diferentes fuentes de nitrógeno D1 (harina de pescado), D2 (pasta de soya) y D3 (urea), estas fueron iso-calóricas con 2900 kilocalorías de energía digestible en materia seca (kcal ED/ $\mathrm{MS}$ ) e iso-proteícas $13 \%$ proteína bruta (PB). Los periodos de incubación utilizados fueron 0, 2, 4, 8, 12, 24, 48 y 72 horas. En la evolución del $\mathrm{pH}$ en la cinética ruminal de las tres dietas con diferentes fuentes de nitrógenos, se puede determinar que la D3 y D1 registraron el mayor $\mathrm{pH}(\mathrm{P}<0.05)$ a las 8 horas de incubación (7.84 y 7.82) respectivamente. La Digestibilidad in vitro de la materia seca (DIVMS) fue superior $(\mathrm{P}<0.05)$ en la D1 entre las 12 y 48 horas de incubación (36.66 y 48.90\%). La degradabilidad inicial o parcial soluble (a), fracción potencialmente degradable (b), degradabilidad potencial y la velocidad de degradación de (b), no fueron significativas $(\mathrm{P}>0.05)$. Este estudio abre una alternativa para la disminución de los costes en la alimentación de rumiantes sobre todo orientado a las dietas de mantenimiento en la estación seca.

Palabras clave: urea, degradación, proteínas, alimentación.
$\mathrm{T}$ he objective of this work was to determine the fermentation kinetics and in vitro ruminal degradability of diets with different nitrogen source for feeding ruminants. Three diets were formulated (D) with different sources of nitrogen D1 (fishmeal), D2 (soybean paste) and D3 (urea), these were iso-caloric with 2900 kilocalories of digestible energy in dry matter (kcal ED / MS ) and iso-proteins 13\% crude protein (PB). The incubation periods used were $0,2,4,8,12,24,48$ and 72 hours. In the evolution of the $\mathrm{pH}$ in the ruminal kinetics of the three diets with different sources of nitrogens, it can be determined that the D3 and D1 recorded the highest $\mathrm{pH}(\mathrm{P}$ $<0.05)$ at 8 hours of incubation (7.84 and 7.82) respectively. The in vitro Digestibility of the dry matter (DIVMS) was higher $(\mathrm{P}<0.05)$ in the D1 between 12 and 48 hours of incubation (36.66 and $48.90 \%$ ). The initial or partial soluble degradability (a), potentially degradable fraction (b), potential degradability and degradation rate of $(\mathrm{b})$, were not significant $(\mathrm{P}>0.05)$. This study opens an alternative for the reduction of costs in the feeding of ruminants especially oriented to maintenance diets in the dry season.

Key words: urea, degradation, proteins, food. 


\section{Introducción}

$\mathrm{L}$ a harina de pescado se ha utilizado tradicionalmente en la alimentación animal, tanto en monogástricos como rumiantes, asi mismo el uso de soja como fuente proteica en alimentación animal, ya sea aprovechando el grano o la torta de soya como fuente de proteína vegetal (Garzón y Navas, 2003). Por otra parte, la urea representa un valioso y económico recurso alimenticio para los rebaños donde la única fuente alimenticia son los forrajes, normalmente deficientes en proteínas. Este elemento provee el nitrógeno requerido para la fermentación ruminal y la formación de proteínas, bajo diversas fórmulas en el concentrado, en el ensilaje y en varios tipos de mezclas (Araque, 2001).

La harina de pescado es una fuente proteica de bastante uso en la preparación de raciones para el consumo animal, rica en aminoácidos esenciales como cisteína, metionina y cistina, los cuales son limitantes sobre todo en monogástricos. Sin embargo, tiene un precio elevado para el productor (Mariño, 2012).

Así mismo, el uso de la soja (Glycine max) en la alimentación animal ha abierto un amplio panorama a la industria de concentrados, al permitir la formulación de dietas con una excelente concentración y disponibilidad de energía, aminoácidos y ácidos grasos esenciales. Por su alto contenido de grasas (18-20\%) y proteínas (37-38\%), el fríjol de soya se presenta como una valiosa materia prima para su utilización en la industria destacándose la extracción de aceites y la formulación de alimentos balanceados para animales (Garzón, 2010). Por otro lado, la urea también es una fuente de nitrógeno para los rumiantes, sin embargo, su uso depende de la habilidad de la flora microbiana del rumen para incorporarla en la formación de sus propios tejidos (Araque, 2001).

Por otra parte, la urea representa un valioso y económico recurso alimenticio para los rebaños donde la única fuente alimenticia son los forrajes, normalmente deficientes en proteínas. Este elemento provee el nitrógeno requerido para la fermentación ruminal y la formación de proteínas, bajo diversas fórmulas en el concentrado, ensilaje y en varios tipos de mezclas (Araque, 2001). Cuando se sustituyen las fuentes nitrógeno de las proteínas formuladas, tanto de la harina de pescado como de la soya, se evidencia que la urea puede sustituir un porcentaje importante de estas fuentes sin detrimento de la respuesta animal (Zapata et al., 2004). La fuente más común de nitrógeno no proteínico (NNP) usada en la alimentación de rumiantes es la urea, debido a su costo bajo y a su equivalente proteínico elevado de $281 \%$. Una unidad de urea en la dieta puede sustituir cinco unidades de harina de soja (Glycine max) (Pinos et al., 2010). Una fuente de NNP aumentaría el espacio para la inclusión de ingredientes en la dieta sustituyendo fuentes de proteína vegetal de costo alto y disponibilidad limitada, mejorando el sincronismo de nutrientes en el rumen sin comprometer el rendimiento animal (Souza et al., 2010). Por el periodo de adaptación requerido de los rumiantes a la urea y con base en la sincronización de la tasa de degradación de nutrientes en el rumen, la digestibilidad de la fibra mejora al usar una fuente de NNP (Ørskov, 1999). Todavía existe un desconocimiento sobre el uso de fuentes de nitrógeno en la alimentación de rumiantes, y es preciso determinar el porcentaje de digestibilidad y su valor nutricional; en una etapa posterior habría que cuantificar el nivel óptimo de sustitución de otras fuentes de proteína de uso frecuente en Ecuador y la elaboración de dietas balanceadas para rumiantes. Solo la provincia de Los Ríos genera anualmente más de 1.5 millones de toneladas métricas de subproductos de arroz, maíz y soya (pancas, hojas, tuzas) que, empleados racionalmente, al enriquecerlos con melaza y urea o conservándolos adecuadamente (henificación, amonificación, ensilaje) pueden constituirse en una alternativa para mejorar su calidad nutritiva y digestibilidad. Además, el uso de estos residuos contribuiría a reducir los costos de alimentación (considerado el rubro más elevado dentro de los costos de producción de las explotaciones pecuarias), evitar la contaminación del medio ambiente, debido a la acumulación a campo abierto y la quema (Sánchez y Zambrano, 2007). Con la inclusión de nitrógeno no proteico (NNP) en las dietas de los rumiantes se ha logrado regularizar y mantener niveles altos de amoniaco, que ha mostrado ser favorable para el desarrollo de la flora ruminal que utiliza el NNP para su multiplicación, mejorándose así la tasa de consumo, degradación y digestión de los alimentos fibrosos, esta mejora en la función ruminal, permite balancear el déficit de proteína e incrementar la eficiencia de utilización de los carbohidratos (Obispo et at. 2001). Por tanto, el objetivo de la presente investigación fue determinar la cinética de fermentación y degradabilidad ruminal in vitro en dietas con diferente fuente de nitrógeno.

\section{Materiales y métodos}

T a investigación se realizó en el laboratorio de Rumiología y Metabolismo Nutricional (RUMEN) de la Universidad Técnica Estatal de Quevedo (UTEQ), provincia de Los Ríos, Ecuador. Se formularon tres dietas con diferentes fuentes de nitrógeno D1 (harina de pescado), D2 (pasta de soja) y D3 (urea), estas fueron Iso-calóricas (2900 kcal ED $/ \mathrm{kg}$ de MS) e Iso-proteicas (13\% PB), en una ración total mezclada (RTM), con otras materias primas energéticas (maíz, arroz, residuo de maíz, y otras) y aditivos de acuerdo a las recomendaciones del National Research Council (NRC, 2001), para bovinos en mantenimiento (Cuadro 1).

Para determinar la degradabilidad ruminal in vitro de la materia seca en cada uno de los tratamientos, se preparó una muestra compuesta con alícuotas de las dietas. Se siguió 
el protocolo recomendado por el fabricante del sistema de incubación DAISY II $®$ (Ankom 2014), usando bolsas filtro ANKOM F-57 (Ankom Technology, Macedon, NY, EUA) con tamaño de poro de $25 \mu \mathrm{m}$ y dimensiones de 5 x $4 \mathrm{~cm}$ fabricadas de poliéster/polietileno con filamentos extruidos en una matriz de tres dimensiones (Giraldo et al., 2007). Se utilizaron dos animales Brahman de $450 \pm 25 \mathrm{~kg}$ de peso vivo, castrados y fistulados en el rumen a los cuales se les extrajo líquido ruminal con un sistema de succión al vacío, en termos aclimatados con agua previamente a $40{ }^{\circ} \mathrm{C}$. Se preparó con anterioridad la solución buffer (saliva artificial) con fosfato de sodio di-básico anhidro $(3.6 \mathrm{~g} / \mathrm{L})$, bicarbonato de sodio $(9.8 \mathrm{~g} / \mathrm{L})$ y cloruros de sodio $(47 \mathrm{~g} / \mathrm{L})$, calcio $(4 \mathrm{~g} / \mathrm{L})$, potasio $(57 \mathrm{~g} / \mathrm{L})$ y magnesio $(6 \mathrm{~g} / \mathrm{L})$, aclimatada en baño maría a $40{ }^{\circ} \mathrm{C}$. Se determino el $\mathrm{pH}$ a la cal, siendo optimo $7 \pm 0.5$, de no cumplir con ese requerimiento se niveló adicionando hidróxido de sodio $(\mathrm{pH}<6.5)$ y/o ácido sulfúrico $(\mathrm{pH}>7.5)$. Se utilizó una relación solución buffer: líquido ruminal (3:2). Previo a la incubación se encendió el sistema ANKOM DAISY II para mantener la temperatura requerida de 40 ${ }^{\circ} \mathrm{C} \pm 0.5$. Esta temperatura y condiciones simulan el estado del rumen, el proceso de mezcla del líquido ruminal $(9.6 \mathrm{~L})$ y solución buffer $(14.4 \mathrm{~L})$ se mantuvo en presencia de $\mathrm{CO}_{2}$ para evitar perdida de los microorganismos anaeróbicos. Para la prueba de digestibilidad in vitro se depositaron $0.5 \mathrm{~g}$ de muestra molida a $2 \mathrm{~mm}$ en el interior de bolsas ANKOM F-57, de acuerdo a la metodología planteada por (ANKOM Technology, 2014). Se incubo el material a 0, 2, 4, 8, 12, 24, 48 y 72 horas, por cada tratamiento se prepararon seis muestras. Finalmente se retiraron las muestras para ser lavadas con agua corriente, y secadas en una estufa Memmert a $65{ }^{\circ} \mathrm{C}$ por 48 horas. Para los cálculos respectivos de degradabilidad in vitro de la materia seca.

Se utilizó un Diseño Completamente al Azar (DCA), cada unidad experimental tuvo una bolsa de degradabilidad F-57 (ANKOM Technology, 2014), se emplearon seis bolsas en cada tiempo de incubación ( $0,2,4,8,24,48,72$ horas) por cada tratamiento (42 bolsitas) con un total de 168 unidades, que fueron incubadas en tres equipos de degradación in vitro DAISY II (ANKOM Technology, 2014). El análisis de datos se realizó mediante el ADEVA y las medias fueron separadas mediante la prueba de Tukey $(\mathrm{P} \leq 0.05)$, con la utilización del paquete estadístico (Statistical Analysis System. Versión 9.0, 2004). Los parámetros de la cinética de degradación se calcularon con el modo de resolución GRG NONLINEAR de la función SOLVER de Microsoft EXCEL®.

Cuadro 1. Dietas con diferentes fuentes de nitrógeno utilizadas en la cinética de fermentación y degradabilidad ruminal in vitro y el análisis calculado

\begin{tabular}{lrrrr}
\hline \multirow{2}{*}{ Ingredientes } & \multicolumn{3}{c}{ Dietas (\%) } & \\
\cline { 2 - 4 } & \multicolumn{1}{c}{ D1 } & \multicolumn{1}{c}{ D2 } & \multicolumn{1}{c}{ D3 } & \\
Maíz seco & 38.30 & 34.88 & 43.90 & \\
Polvillo de cono & 25.00 & 25.00 & 25.00 & \\
Harina pescado exportación & $\mathbf{7 . 7 0}$ & & & \\
Pasta de soja & & $\mathbf{1 0 . 3 2}$ & & \\
Urea & & & $\mathbf{1 . 3 0}$ & \\
Residuo de maíz & 25.00 & 25.00 & 25.00 & \\
Premix Broiler & 0.20 & 0.20 & 0.20 & \\
Carbonato de calcio & 1.80 & 1.80 & 1.80 & \\
Fosfato Di cálcico & 0.50 & 1.30 & 1.30 & \\
Sal & 1.50 & 1.50 & 1.50 & \\
\hline Total & $\mathbf{1 0 0 . 0 0}$ & $\mathbf{1 0 0 . 0 0}$ & $\mathbf{1 0 0 . 0 0}$ & \\
\hline Análisis calculado & $\mathbf{D 1}$ & $\mathbf{D 2}$ & $\mathbf{D 3}$ & Requerimiento \\
\hline Proteína total (\% MS) & 12.18 & 12.18 & 12.18 & $12.00-14.00$ \\
Energía metabolizable (kcal) & 2889.21 & 2812.20 & 2891.30 & $2800-3000$ \\
Calcio (\%) & 1.00 & 1.02 & 1.00 & \\
Fósforo disponible (\%) & 0.35 & 0.36 & 0.34 & $0.30-0.40$ \\
Fibra (\%) & 12.15 & 12.66 & 12.22 & $9.00-17.00$ \\
\hline An & & &
\end{tabular}

Análisis calculados según requerimientos NRC 2001 


\section{Resultados y discusión}

L a evolución del $\mathrm{pH}$ en la cinética ruminal de las tres

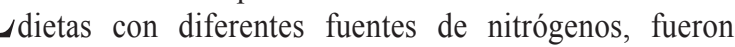
similares (Cuadro 2). Bruni y Chilibroste (2001) consideran que los parámetros de la cinética de fermentación describen la digestión y caracterizan propiedades intrínsecas del alimento que limitan su disponibilidad para el rumiante, determinan la proporción de nutrientes consumidos que pueden ser absorbidos y utilizados por el animal, y dependen de un activo crecimiento y desarrollo de la población microbiana del rumen. Así, la degradabilidad de las tres dietas fueron similares en todos los tiempos de fermentación. Estos resultados concuerdan con Araujo (2005) quien menciona que los factores pueden modificar la digestibilidad y se pueden clasificar en dos tipos, los factores dietarios y los factores animales, ya que el aumento del nivel de ingestión causa una velocidad de tránsito intestinal y hay disminución del tiempo de acción enzimático, que puede disminuir levemente la digestibilidad (1 a 3\%). Respecto a la digestibilidad in vitro de la materia seca (Cuadro 3), se pueden observar que los mayores porcentajes $(\mathrm{p}<0.05)$ fueron para la D1 a las 12 y 48 horas de incubación (36.66 y $48.90 \%$ ). La degradabilidad inicial o porción soluble (a), fracción potencialmente degradable (b), degradabilidad potencial y la velocidad de degradación de $b$, no fueron significativas $(p>0.05)$. Esto puede deberse a los posibles cambios de la flora celulítica ruminal como consecuencia de la administración de altas proporciones de concentrado en la ración. Resultados similares a los encontrados por Bochi et al. (1999); Carro et al. (1999) quienes no encontraron diferencias en la digestibilidad tanto in vivo como in vitro al

Cuadro 2. Evolución del pH en tres dietas con diferente fuente de nitrógeno en ocho periodos de incubación

\begin{tabular}{crrrrrr}
\hline \multirow{2}{*}{$\begin{array}{c}\text { Periodos de } \\
\text { incubación } \\
\text { (h) }\end{array}$} & \multicolumn{2}{c}{$\begin{array}{c}\text { Dietas con diferentes } \\
\text { fuente de nitrógeno }\end{array}$} & EEM & P<Trat \\
\cline { 2 - 4 } & $\mathbf{D 1}$ & $\mathbf{D 2}$ & $\mathbf{D 3}$ & & \\
\hline 0 & $8.32 \mathrm{a}$ & $8.30 \mathrm{a}$ & $8.31 \mathrm{a}$ & 0.010 & 0.784 \\
2 & $7.93 \mathrm{a}$ & $8.02 \mathrm{a}$ & $8.01 \mathrm{a}$ & 0.030 & 0.493 \\
4 & $7.97 \mathrm{a}$ & $7.87 \mathrm{a}$ & $7.93 \mathrm{a}$ & 0.023 & 0.230 \\
8 & $7.82 \mathrm{a}$ & $7.71 \mathrm{~b}$ & $7.84 \mathrm{a}$ & 0.011 & 0.001 \\
12 & $7.47 \mathrm{a}$ & $7.44 \mathrm{a}$ & $7.62 \mathrm{a}$ & 0.010 & 0.000 \\
24 & $7.31 \mathrm{a}$ & $7.34 \mathrm{a}$ & $7.34 \mathrm{a}$ & 0.007 & 0.150 \\
48 & $7.16 \mathrm{a}$ & $7.14 \mathrm{a}$ & $7.14 \mathrm{a}$ & 0.008 & 0.571 \\
72 & $7.19 \mathrm{a}$ & $7.18 \mathrm{a}$ & $7.17 \mathrm{a}$ & 0.007 & 0.530 \\
\hline
\end{tabular}

D1=Harina de pescado, D2=Torta Soja, D3=Urea, EEM=Error estándar de la media. Medias con una letra común, no son significativamente diferentes (Tukey, $\mathrm{p}>0.05$ ) evaluar 11 forrajes.

Por otra parte, los forrajes y raciones con concentraciones similares pueden variar en su perfil o en los parámetros ( $\mathrm{a}, \mathrm{b}$ y c) de degradabilidad ruminal, debido a factores asociados entre nutrientes y a la conformación o arreglo de estos en cada ingrediente (Liu et al., 2002). En este caso la concentración de nutrientes en las raciones no fue diferente entre dietas ni tampoco los volúmenes indicando que tienen un comportamiento de degradabilidad similar, aunque el origen de los nutrientes en la ración haya variado al incluir las fuentes de nitrógeno en cada una de ellas. Esto coincide con lo reportado por Cone y Van Gelder (2000), quienes mencionaron que la proteína no es bien fermentada, se incrementa la producción de amoniaco, y se reduce el volumen de la fermentación ruminal.

Cuadro 3. Degradabilidad in vitro y parámetros cinética ruminal de la MS de tres dietas con diferente fuente de nitrógeno en ocho periodos de incubación

\begin{tabular}{cccccc}
\hline \multirow{2}{*}{$\begin{array}{c}\text { Periodos de } \\
\text { incubación } \\
\text { (h) }\end{array}$} & $\begin{array}{c}\text { Dietas con diferente fuente de } \\
\text { nitrógeno }\end{array}$ & EEM P<Trat \\
\cline { 2 - 6 } & D1 & D2 & D3 & & \\
\hline 0 & $21.57 \mathrm{a}$ & $20.50 \mathrm{a}$ & $19.99 \mathrm{a}$ & 0.4 & 0.34 \\
2 & $23.28 \mathrm{a}$ & $21.93 \mathrm{a}$ & $22.29 \mathrm{a}$ & 0.37 & 0.38 \\
4 & $24.64 \mathrm{a}$ & $24.82 \mathrm{a}$ & $28.56 \mathrm{a}$ & 0.28 & 0.15 \\
8 & $29.11 \mathrm{a}$ & $26.99 \mathrm{a}$ & $27.76 \mathrm{a}$ & 0.42 & 0.19 \\
12 & $36.66 \mathrm{a}$ & $29.31 \mathrm{~b}$ & $30.73 \mathrm{ab}$ & 0.93 & 0.03 \\
24 & $40.40 \mathrm{a}$ & $38.90 \mathrm{a}$ & $40.70 \mathrm{a}$ & 0.46 & 0.30 \\
48 & $48.90 \mathrm{a}$ & $43.86 \mathrm{~b}$ & $44.79 \mathrm{ab}$ & 0.57 & 0.01 \\
72 & $54.11 \mathrm{a}$ & $53.21 \mathrm{a}$ & $51.87 \mathrm{a}$ & 0.67 & 0.45 \\
\hline
\end{tabular}

D1 = Harina de pescado, D2= Torta Soja, D3= Urea, EEM= Error estándar de la media. EEM: a: fracción soluble; b: fracción potencialmente degradable; kd: Tasa degradación hora (\%); c: tasa de pasaje (\% hora). Medias con una letra común, no son significativamente diferentes (Tukey, $\mathrm{p}>0.05$ )

Cuadro 4. Cinética de degradación ruminal de la MS de tres dietas con diferente fuente de nitrógeno en ocho periodos de incubación

\begin{tabular}{crrrrr}
\hline \multirow{2}{*}{ Fracciones } & \multicolumn{6}{c}{ Parámetros de cinética ruminal } \\
\cline { 2 - 6 } & \multicolumn{1}{c}{ D1 } & \multicolumn{1}{c}{ D2 } & \multicolumn{1}{c}{ D3 } & EEM & P<Trat \\
\hline $\mathrm{a}$ & $20.42 \mathrm{a}$ & $20.13 \mathrm{a}$ & $20.96 \mathrm{a}$ & 0.32 & 0.604 \\
$\mathrm{~b}$ & $37.12 \mathrm{a}$ & $36.96 \mathrm{a}$ & $40.44 \mathrm{a}$ & 1.74 & 0.686 \\
$\mathrm{k}_{\mathrm{d}}$ & $0.03 \mathrm{a}$ & $0.03 \mathrm{a}$ & $0.02 \mathrm{a}$ & 0 & 0.934 \\
$\mathrm{c}$ & $21.51 \mathrm{a}$ & $32.86 \mathrm{a}$ & $56.12 \mathrm{a}$ & 4.53 & 0.375 \\
\hline
\end{tabular}

D1=Harina de pescado, D2=Torta Soja, D3=Urea, EEM=Error estándar de la media. a: fracción soluble; b: fracción potencialmente degradable; kd: Tasa degradación hora (\%); c: tasa de pasaje (\% hora). Medias con una letra común, no son significativamente diferentes (Tukey, $\mathrm{p}>0.05$ ) 


\section{Conclusiones}

$\mathrm{L}$ as dietas con diferentes fuentes de nitrógeno; harina de pescado, pasta de soja y urea; pueden ser utilizadas en las raciones como ingredientes, ya que la adición de estas a las dietas no mostraron diferencias en la cinética de fermentación y en la degradabilidad ruminal in vitro de la MS. La inclusión de fuentes de nitrógeno animal, vegetal y química en las dietas genera una disminución en la concentración del pH. Aunque sí se obtiene una mayor Degradabilidad de la MS en la dieta con harina de pescado en los tiempos de incubación de 12 y 48 horas respectivamente.

\section{Agradecimiento}

\begin{abstract}
A la Universidad Técnica Estatal de Quevedo (UTEQ) Apor su financiamiento a través del Fondo Competitivo de Investigación Ciencia y Tecnología (FOCICYT-2016). "Caracterización de ensilajes de pastos tropicales con niveles de inclusión de residuos agrícolas y agroindustriales de uso alimenticio en rumiantes".
\end{abstract}

\section{Bibliografía}

ANKOM Technology. (2014). In vitro True Digestibility with DAISY II Incubator. ANKOM Technology, Macedon, NY.

Araque, C. (2001). La urea en la alimentación de rumiantes. http://www.produccionanimal.com.ar/informacion tecnica/suplementacion_proteica_y_con_nitrogeno_no_ proteico/00-suplementacion_proteica_y_con_nitrogeno_ no proteico.htm

Araujo, O. 2005. Factores que afectan el consumo voluntario en bovinos a pastoreo en condiciones tropicales. IX Seminario de pastos y forrajes. Departamento de Zootecnica. Facultad de Agronomía. Universidad de Zulia. Venezuela.

Bruni, M., Chilibroste, P. (2001). Simulación de la digestión ruminal por el método de la producción de gas. Archivo Latinoamericano Producción Animal. 9: 43-51.

Bochi, O., Carro, M., Valdés, C., González, J., López, S. (1999). Digestibilidad in vitro de forrajes y concentrados. Efecto de la ración de los animales donantes de líquido ruminal. Archivos de Zootecnia, 48(181): 51-61
Carro, M., López, S., Valdéz, C., Ranilla, M. (1999). Efecto de la suplementación nitrogenada sobre la fermentación ruminal in vitro de forrajes deficientes en nitrógeno, Archivos de zootecnia, 48(183): 295-306.

Cone, JW., Van Gelder, AH. (2000). Influence of protein fermentation on gas production. In: Gas Production: Fermentation Kinetics for Feed Evaluation and to Assess Microbial Activity. An EAAP Satellite Symposium, British Society of Animal Science and Wageningen University, Wageningen, The Netherlands, pp. 23-24.

Garzón, A., Navas, E. (2003). Características nutricionales de fuentes alimenticias y su utilización en la elaboración de dietas para animales domésticos. Boletín técnico No.38. CORPOICA. Pronatta Villavicencio, $47 \mathrm{p}$.

Giraldo, L., Gutiérrez, L., Rúa, C. (2007). Comparación de dos técnicas in vitro e in situ para estimar la digestibilidad verdadera en varios forrajes tropicales. Rev. Col. Cienc. Pec. 20: 269-279.

Liu, J., Nissim, D., Thomas, JK. (2002). Equity Valuation Using Multiples. Journal of Accounting Research 40, $135-172$.

NRC. (2001). National Research Council. Nutrient requeriments of dairy cattle. $6^{\mathrm{a}}$ Revised Edition. National Academy Press. Washington,D.C.

Ørskov, ER. (1999). Supplement strategies for ruminants and management of feeding to maximize utilization of roughages. Preventive Vet. Med. 38: 179-185.

Obispo, NE., Pares, P., Hidalgo, C., Palma, J., Godoy, S. (2001). Consumo de forraje y ganancia diaria de peso en bovinos de carne en crecimiento suplementados con fuentes proteicas. Zootecnia Tropical. 19:423-442.

Pinos, J., Peña, J., González, S., Bárcenas, R., Salem, A. (2010). Effects of a slow-release coated urea product on growth performance and ruminal fermentation in beef steers. Italian J. Anim. Sci. 9: 16-19.

Sánchez, A., Zambrano, D. (2007). Valoración nutritive de los principals subproductos agrícolas para alimentación de ovinos tropicales en la parte alta de la Cuenca del río Guayas. Boletín técnico de la Universidad Técnica Estatal de Quevedo. No. 012.

SAS. (2001). Institute Inc., SAS/STAT; Software Version 9.00. Cary, NC, USA. https://support.sas.com/documentation/ onlinedoc/91pdf/sasdoc.../stat_ug_7313.pdf.

Souza, V., Almeida, R., Silva, P., Pierlasky, C., Jesus, P., Pereira, M. (2010). Substituição parcial de farelo de soja por ureia protegida na produção e composição do leite. Arq. Bras. Med. Vet. Zootec. 62: 1415-1422. 